\title{
Effects of source and receiver locations in predicting room transfer functions by a phased beam tracing method
}

Jeong, Cheol-Ho; Ih, Jeong-Guon

Published in:

Acoustical Society of America. Journal

Link to article, DOI:

$10.1121 / 1.3699268$

Publication date:

2012

Document Version

Early version, also known as pre-print

Link back to DTU Orbit

Citation (APA):

Jeong, C-H., \& Ih, J-G. (2012). Effects of source and receiver locations in predicting room transfer functions by a phased beam tracing method. Acoustical Society of America. Journal, 131(5), 3864-3875.

https://doi.org/10.1121/1.3699268

\section{General rights}

Copyright and moral rights for the publications made accessible in the public portal are retained by the authors and/or other copyright owners and it is a condition of accessing publications that users recognise and abide by the legal requirements associated with these rights.

- Users may download and print one copy of any publication from the public portal for the purpose of private study or research.

- You may not further distribute the material or use it for any profit-making activity or commercial gain

- You may freely distribute the URL identifying the publication in the public portal

If you believe that this document breaches copyright please contact us providing details, and we will remove access to the work immediately and investigate your claim. 


\title{
Effects of source and receiver locations in predicting room transfer functions by a phased beam tracing method
}

\author{
Cheol-Ho Jeong ${ }^{\text {a) }}$ \\ Acoustic Technology, Department of Electrical Engineering, Technical University of Denmark, DK-2800, \\ Kongens Lyngby, Denmark \\ Jeong-Guon Ih \\ Center for Noise and Vibration Control (NOVIC), Department of Mechanical Engineering, Korea Advanced \\ Institute of Science and Technology, 305-701 Daejeon, Korea
}

(Received 30 November 2011; revised 13 March 2012; accepted 13 March 2012)

\begin{abstract}
The accuracy of a phased beam tracing method in predicting transfer functions is investigated with a special focus on the positions of the source and receiver. Simulated transfer functions for various source-receiver pairs using the phased beam tracing method were compared with analytical Green's functions and boundary element solutions up to the Schroeder frequency in simple rectangular rooms with different aspect ratios and absorptions. Only specular reflections were assumed and diffraction was neglected. Three types of error definitions were used: average error level over a narrow band spectrum, average error level over a 1/3 octave band spectrum, and dissimilarity measure. The narrow band error and dissimilarity increased with the source-to-receiver distance but converged to a certain value as the reverberant field became dominant. The 1/3 octave band error was found to be less dependent on the source-receiver distance. The errors are increased as the aspect ratio becomes more disproportionate. By changing the wall absorption from 0.2 to 0.8 for a rectangular room, the average narrow and $1 / 3$ octave band error are deviated by around $1.5 \mathrm{~dB}$. A realistic nonuniform distribution of the absorption increases the error, which might be ascribed to wave phenomena evoked by the impedance-discontinuous boundary. (C) 2012 Acoustical Society of America. [http://dx.doi.org/10.1121/1.3699268]
\end{abstract}

PACS number(s): 43.55.Ka [NX]

Pages: 3864-3875

\section{INTRODUCTION}

Phased geometrical acoustics methods are known to have obvious advantages over the other methods at medium frequencies as they are faster than wave-based methods and more accurate than energy-based methods. ${ }^{1-3}$ At low frequencies, wave based methods are, with no doubt, the most reliable and appropriate tools in calculating transfer functions. At frequencies well above the Schroeder frequency, room modes are highly overlapped, and therefore individual modal characters do not need to be taken into account. At such high frequencies, the geometrical acoustics methods are approximate but reliable and fast prediction schemes. However, at around the Schroeder frequency both wave based and geometrical acoustics methods cannot tackle acoustic problems properly: The wave based methods require a lot of computational expenditure due to a huge number of elements, whereas lack of phase information and modal characteristics leads the geometrical acoustics methods to inaccurate outcomes. Therefore the geometrical acoustics methods retaining phase have been suggested as alternatives with a relatively short calculation time; these methods can account for interference that is still important at medium frequencies. However, the phased geometrical acoustics is still an approximate model based on the geometrical tracing,

\footnotetext{
a) Author to whom correspondence should be addressed. Electronic mail: chj@elektro.dtu.dk
}

therefore certain systematic errors can happen in predicting room acoustics. This paper aims to investigate such systematic errors of a phased beam tracing method at low frequencies with a special focus on the source-to-receiver distance.

The accuracy of the phased geometrical acoustics methods over the conventional geometrical acoustics methods is well documented. Suh and Nelson proved that a phased image source method can account for wave interference well, whereas conventional geometrical simulations cannot model interference. ${ }^{1}$ Significantly reduced errors in simulated impulse responses were observed for the phased image source method. Lam discussed the accuracy of a phased image source method employing plane and spherical wave reflection coefficients in comparisons with boundary element simulations and energy based geometrical method simulations in the time and frequency domain. ${ }^{2}$ The plane wave reflection model was found to have noticeable errors at higher admittance values and at longer delay time, but the accuracy improves as the frequency increases. Jeong et al. examined another reflection modeling that takes into account finite boundary surfaces and angle dependence in a phased beam tracing method (PBTM), resulting in a notable improvement in the early parts of impulse responses. ${ }^{3}$

The main focus of this study is to investigate how the simulation error changes with the source-to-receiver distance in simple rectangular enclosures and to eventually quantify the upper limit of the PBTM error in such rooms. Because the simulation error is amplified near off-resonance frequencies 
that change with the source and receiver locations, the simulation error is related to the position of the source and receiver. In general, the error is small for a short source-to-receiver distance, whereas the error is likely to be amplified for a long distance. The simulation error is also ascribed to the scanning error because the phased beam tracing used does not incorporate a beam-splitting algorithm. The longer the travel distance, the larger the scanning error due to the non-splitting algorithm. Therefore the main concerns are how large the simulation error becomes with the non-splitting algorithm, and how the simulation error changes with the source-to-receiver distance $\left(\mathrm{d}_{\mathrm{SR}}\right)$, when simulating rectangular rooms where diffraction and diffuse reflections are negligible.

The total transfer function predicted by PBTM consists of the direct field and reverberant field. The reverberant energy is mainly determined by the absorption of the system, e.g., the equivalent absorption area of the room and air absorption. In a diffuse sound field, the reverberant energy is constant regardless of the distance between the source and receiver, whereas the direct energy decreases with increasing $\mathrm{d}_{\mathrm{SR}}$. Their energy ratio is defined as the direct-to-reverberant (D-R) energy ratio.

The D-R energy ratio is mainly associated with two factors: room absorption and $\mathrm{d}_{\mathrm{SR}}$. If $\mathrm{d}_{\mathrm{SR}}$ is short enough, the D-R energy ratio tends to be high because the direct field will dominate near the point of excitation. If the room boundaries are acoustically absorptive, there are substantial attenuations of the reflections from the boundaries, producing a high D-R energy ratio. All in all, in moderately damped large rooms, the direct sound and early reflections are dominant contributers to the steady-state pressure, ${ }^{4,5}$ resulting in more accurate results because PBTM results are accurate particularly in the early parts of impulse responses. ${ }^{3,4}$ Such a D-R energy ratio is also related to one of the important underlying assumptions of the statistical energy analysis, that is, high modal overlap. The modal overlap factor of a room is $27.6 \mathrm{~V} \cdot f^{2} /\left(T_{60} c_{o}^{3}\right)$, ${ }^{6}$ where $V$ is the volume, $f$ is the frequency, $T_{60}$ is the reverberation time, and $c_{o}$ is the speed of sound. The modal overlap factor is proportional to the volume and absorption of the three-dimensional (3D) system. Therefore reasonably large rooms and/or highly damped rooms are also good application examples of all the high frequency energy methods.

Transfer functions in a room have numerous poles and zeros. The poles that are determined by the eigenvalues are independent of the source and observation point. The zeros, however, are dependent on their locations. Therefore changes in either the source or receiver position inevitably lead to changes in the locations and magnitudes of the zeros as well as the D-R energy ratio. Because PBTM and any geometrical acoustics methods become less accurate in calculating late reflections, a favorable condition for PBTM to calculate transfer functions is a high D-R ratio or high earlyto-late energy ratio. Inherent simulation errors arise mostly in late reflections due to incorrect scanning, spatial aliasing problems, and limited reflection numbers. Lam discussed such a simulation error and named it as $-30 \mathrm{~dB}$ cutoff limit for the geometrical contributions because the minima of predicted transfer functions are unlikely to reach below $-30 \mathrm{~dB}$ from the maxima. ${ }^{2}$ A brief introduction to the simulation error will be given in Sec. II B.

This study is mainly concerned with effects of $d_{S R}$ on the accuracy of PBTM. First, a reasonably low absorption coefficient of 0.1 is assumed as a boundary condition of three rectangular parallelepiped rooms with different aspect ratios, and PBTM results are compared with acoustic Green's functions. Second, only a proportionate rectangular room is chosen as a scaled lecture room for which PBTM simulations are compared with boundary element simulations for various absorption cases. Six uniform absorptions and one non-uniform distribution of the wall absorption are simulated and compared. Three error measures are defined to examine the PBTM error in terms of $\mathrm{d}_{\mathrm{SR}}$.

\section{PHASED BEAM TRACING AND ITS SIMULATION ERROR}

\section{A. Phased beam tracing}

The basic idea of the phased geometrical acoustics is to retain phase information to account for wave phenomena, particularly interference. As a pioneering attempt, phase was introduced into a ray-tracing model so that it could be applied to low to medium frequencies. ${ }^{7}$ Phased image source models have been developed later and validated with measurements. ${ }^{1,2}$ Wareing and Hodgson developed a transfer-matrix model integrated into a beam tracing method for multilayered surfaces for accurate boundary modeling. ${ }^{8}$ An adaptive beam tracing method incorporating the uniform theory of dfractinon ${ }^{9}$ was tested in Bell labs by Tsingos et al., yielding a remarkable agreement with measurements. ${ }^{10}$

Inclusion of phase is twofold: phase on reflections from surfaces and propagation phase. The former phase requires complex reflection coefficients at the boundary surfaces; this is practically not always the case. The latter, however, is quite simply implemented by knowing the traveling distance from the source to receiver $\left(d_{\text {tavel }}\right)$ and taking into account a term, $\exp \left(-j k d_{\text {tavel }}\right)$, in calculation of sound pressures. In this study, the propagation phase is always included, but the reflection phase is omitted due to the use of real-valued absorption coefficients and real-valued surface impedances as input data. No matter the reflection coefficient is complex-valued, PBTM can estimate the sound pressures at receiver locations with help of the propagation phase.

PBTM used in this study is based on the triangular beam tracing algorithm by Lewers, ${ }^{11}$ but extended to include phase. The tracing algorithm consists of source generation, surface-geometry definition, traces of beams, and receiver detection. Source division is based on an icosahedron, which makes the beam cross section an equilateral triangle. Then all edges of the equilateral triangles of the icosahedron are divided into $p$ equal lengths, resulting in a polygon with $20 \cdot p^{2}$ triangular faces. For each receiver, the source is rotated so that a triangle faces toward the receiver in order not to miss the direct sound. Room boundary surfaces should be planar; they are mathematically modeled as $A_{i} x+B_{i} y+C_{i} z+D_{i}=0$. A trajectory of a beam is scanned by a combined process of determining the nearest plane, finding the new image source, and calculating the reflected 
vector. A beam is defined by a central axis and three boundary planes, each plane forming a side of the beam. Beams do not fragment on reflection, and the direction after reflection is determined entirely by its central axis. Once the trajectory of the beam is identified, the possibility that a point receiver is surrounded by the beam boundary planes is tested using the normal vectors of the boundary walls. Following a positive receiver point test, the complex pressure for the beam undergoing the reflection path is calculated and finally the transfer function is constructed.

Therefore a PBTM result is a summation of the contributions of the emitted beams from a source, which hit a receiver in a room. Assuming a simple source emitting spherical waves, the free-space Green's function, ${ }^{12}$ $A \cdot \exp (-j k r) / r$, is a basis to calculate the transfer function at an observation point, where $k$ is the wavenumber, $j$ is the imaginary unit, $r$ is the distance from the corresponding source to the observation point, and $A$ is an arbitrary constant. For each reflection, a reflection coefficient is multiplied to the free-space Green's function. For example, if the boundary condition is given in terms of an absorption coefficient $\left(\alpha_{i}\right)$, the simplest reflection coefficient is calculated to $\sqrt{1-\alpha_{i}}$, assuming an acoustically hard wall with zero phase shift. If a specific surface impedance $(\zeta)$ is known, the plane wave reflection coefficients for an infinitely large panel of $(\zeta \cos \theta-1) /(\zeta \cos \theta+1)$ can be used. Complex reflection coefficients can improve its accuracy, particularly when the phase shifts at walls are quite severe. For most reflective surfaces, the use of real-valued reflection coefficients are proved to be quite acceptable, ${ }^{1,2}$ because the phase shift on reflection is quite negligible.

However, challenges still remain, particularly in considering diffraction and diffuse reflection. In a simply shaped room, one can ignore diffraction, according to Pierce, "amplitudes of the diffracted field usually much weaker than direct and even reflected contributions." 13 Diffraction was only considered in shadow regions in several previous works, assuming its contribution is relatively small in illuminated regions where direct and reflected contributions from a source also reach a listener. ${ }^{10,14}$ On the other hand, Torres et al. claimed that diffraction can be perceived in illuminated regions. ${ }^{15}$ It is still controversial if diffraction should be included for the entire enclosed sound field. As can be seen in Sec. IV, diffraction is not crucial, at least, at low frequencies in the rectangular rooms tested.

Another main problem of PBTM occurs when beams intersect more than one surface. ${ }^{8,11,16-18}$ If an intersecting polygon is detected, there are two solutions: The original beam is followed by its central axis ray ${ }^{3,8,11}$ or the original beam can be split. ${ }^{16,17}$ Beam-splitting algorithms make simulations more accurate, but they become computationally voracious. The phased beam tracing model used incorporates neither splitting algorithms nor diffraction schemes.

\section{B. Simulation errors}

As Lam pointed out, phased geometrical acoustics methods suffer from prediction errors near zeros. ${ }^{2}$ To demonstrate such errors, a unit cube with a source and receiver position at $(0.5,0.5,0.5)$ and $(1,1,0)$, respectively, [all in $\mathrm{m}$ in what follows] is chosen. Three uniform absorption cases of $1 \%$, $5 \%$, and $10 \%$ are simulated by PBTM using 8000 beams up to the 200th reflection orders. To demonstrate how the PBTM simulation changes with the wall absorption, a finite element simulation under a perfectly rigid boundary condition $^{19}$ is also shown in Fig. 1. Note that the inclusion of the rigid boundary condition is not intended to compare the two different simulation models but to illustrate how the peak levels are attenuated and the peaks and troughs are shifted toward low frequency as a natural consequence of the additional absorption.

For the very low absorption case in Fig. 1(a), the simulated pressures at the off-resonance frequencies are quite noisy and inaccurate, e.g., overestimations at frequencies from 20 to $300 \mathrm{~Hz}$, whereas the $10 \%$ absorption case shows more stable results in the same frequency range. As the absorption increases, the responses become less influenced by the simulation noise. The level differences between the pressure maxima and minima in the PBTM predictions for the $1 \%$ and $10 \%$ absorption are 31 and $22 \mathrm{~dB}$, respectively, which concurs with Lam's observation. However, the transfer function for the $1 \%$ absorption case is unacceptably noisy for levels below $-10 \mathrm{~dB}$ from the maximum peak level, whereas the result for the $10 \%$ absorption is not even below $-20 \mathrm{~dB}$ from the peak level.

The absolute level of the simulation error due to the incorrect geometrical tracing is likely to be independent of the frequency. However, the signal to noise ratio is important. When the true level of the response is much higher than the simulation error, the error is not noticeable, e.g., at resonance frequencies. When the true sound pressure becomes smaller than the simulation noise, they start to be noticeable, which happens mostly near off-resonance frequencies. The simulation noise originates from the incorrect beam paths (the sampling error due to the non-splitting algorithm) and incorrect detection for the late reflections, which are mostly related to uncertainties in the late reverberant energy. An increase in the wall absorption leads to an increase in the $\mathrm{D}-\mathrm{R}$ energy ratio by decreasing the uncertainty in the late reverberant energy.

The amount of absorption in the room also affects the convergence of the cumulative pressure. The convergence becomes slow for low absorption cases. In Fig. 2, cumulative pressures as a function of the number of the beams hitting the receiver are shown at two frequencies of $119 \mathrm{~Hz}$ (an offresonance frequency) and $968 \mathrm{~Hz}$ (a resonance frequency) for the two absorption coefficients of $1 \%$ and $5 \%$. The convergence is slowest at the off-resonance frequency for the $1 \%$ absorption in Fig. 2(a), whereas the sound pressure converges faster for the $5 \%$ absorption at the same frequency. The inaccurate responses at the off resonances in Fig. 1(a) probably result from the unconverged pressures. At the resonance frequency of $968 \mathrm{~Hz}$, constructive interference occurs by adding up reflected components from the walls in Figs. 2(b) and 2(d), and their convergence is faster. In general, as the frequency increases, the D-R energy ratio is likely to increase due to increased boundary absorption, for example, classroom ceilings, upholstered chairs, curtains, 

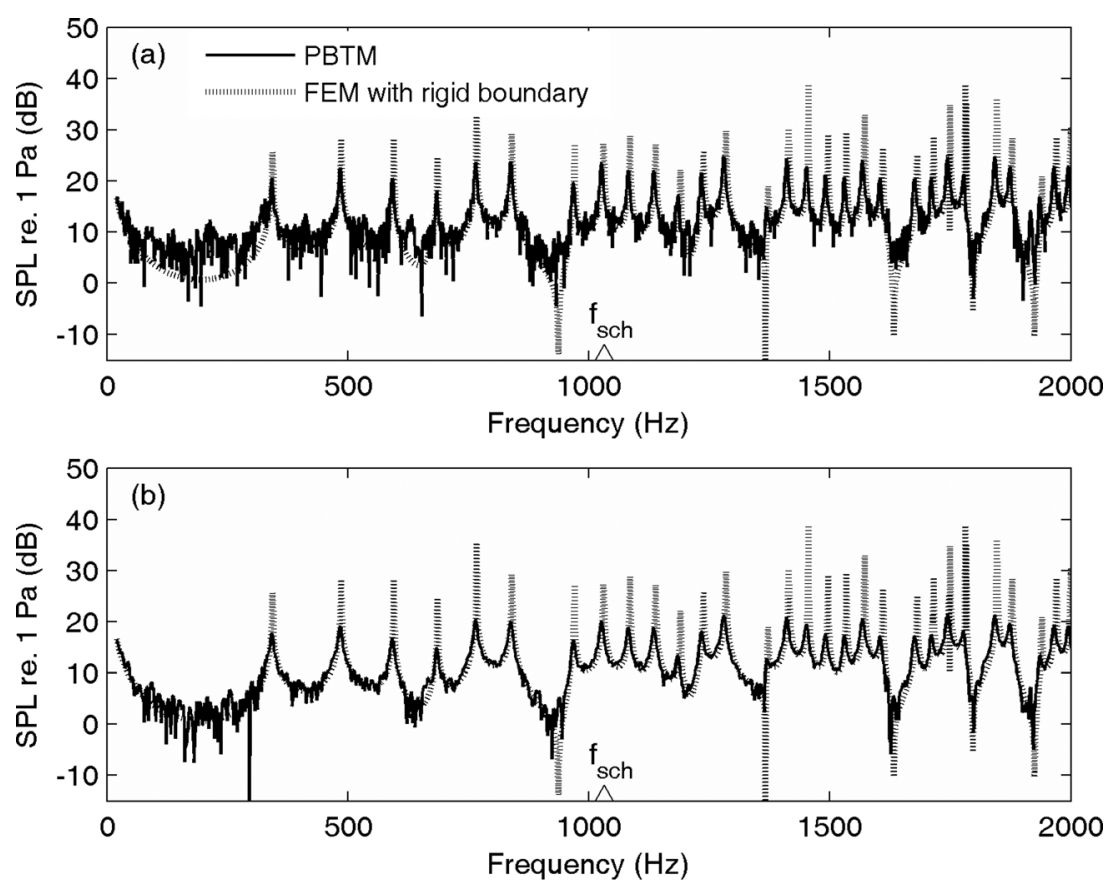

FIG. 1. Comparisons of transfer functions between FEM and PBTM for three absorption cases when a source and receiver are at $(0.5,0.5,0.5)$ and $(1,1$, $0)$, respectively, in a unit cube. The symbol $\triangle$ indicates the Schroder frequency of the room, $1033 \mathrm{~Hz}$. (a) $\alpha=0.01$, (b) $\alpha=0.05$, (c) $\alpha=0.1$.

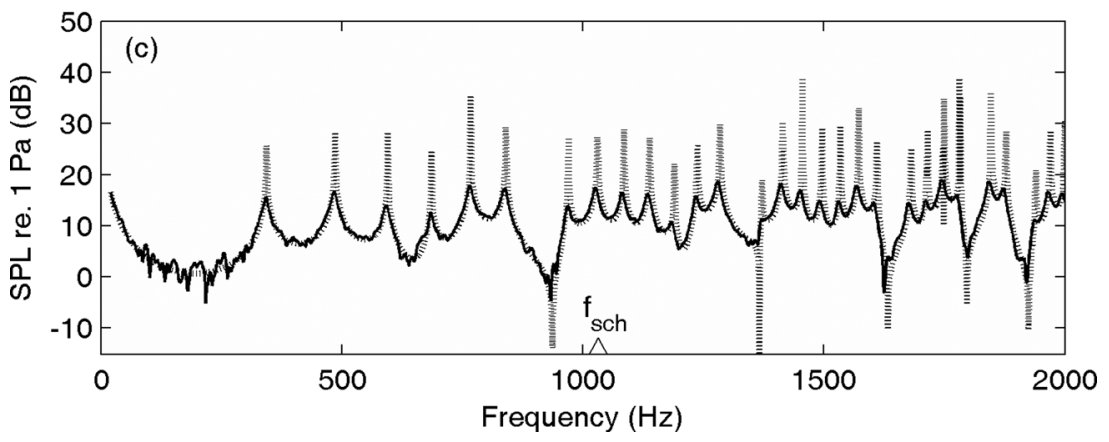

and human bodies can absorb sound more effectively at high frequencies. Consequently the convergence is likely to be faster at higher frequencies.

\section{METHOD}

To quantify the sole influence of the source-to-receiver distance on PBTM simulations, the boundary absorption coefficient is fixed to 0.1 . For this purpose, PBTM simulations are compared with the corresponding Green's functions for three rectangular rooms with different aspect ratios. Absorption coefficients lower than 0.1 are not practical in many actual cases (except for very reverberant sound fields) and may yield quite noisy responses. Absorption coefficients higher than 0.1 may also be problematic in such comparisons because acoustic Green's functions can approximate lightly damped sound fields by a damping term but still using three cosine terms. High absorption cases are actually unrealistic at low frequencies, say below the Schroeder frequency.

To investigate the combined effects of the source-to-receiver distance and boundary absorption, a proportionate room is chosen to be simulated with different absorption cases. For validating PBTM predictions with changing absorption coefficients, boundary element solutions are compared. A realistic non-uniform absorption condition is also included in the comparison.

\section{A. PBTM and acoustic Green's function}

In the PBTM used in this study, a source is assumed to produce a sound pressure of $1 \mathrm{~Pa}$ at $1 \mathrm{~m}$ from a point source in a free field. Therefore the free space Green's function for a harmonic wave is given by

$$
G_{\text {free }}(r)=\frac{1}{r} \exp (-j k r)
$$

To compare transfer functions calculated by PBTM with acoustic Green's functions, one should find a proper volume velocity that produces $1 \mathrm{~Pa}$ at $1 \mathrm{~m}$ from the source. The sound pressure by a monopole at a distance $r$ is given by

$$
p_{\text {mono }}(r)=j \rho c_{o} u_{a} \frac{a}{r} k a \exp (-j k r),
$$

where $\rho$ is the density of air, $u_{a}$ is the velocity at $r=a$, and $a$ is the radius of the monopole. To have an absolute sound pressure of $1 \mathrm{~Pa}$ at $1 \mathrm{~m}$, the source velocity, $u_{a}$, should be

$$
u_{a}=1 /\left(\rho \omega a^{2}\right)
$$

The acoustic Green's function is the solution to the inhomogeneous wave equation with a mass injection source term and given boundary condition as follows: ${ }^{12}$ 

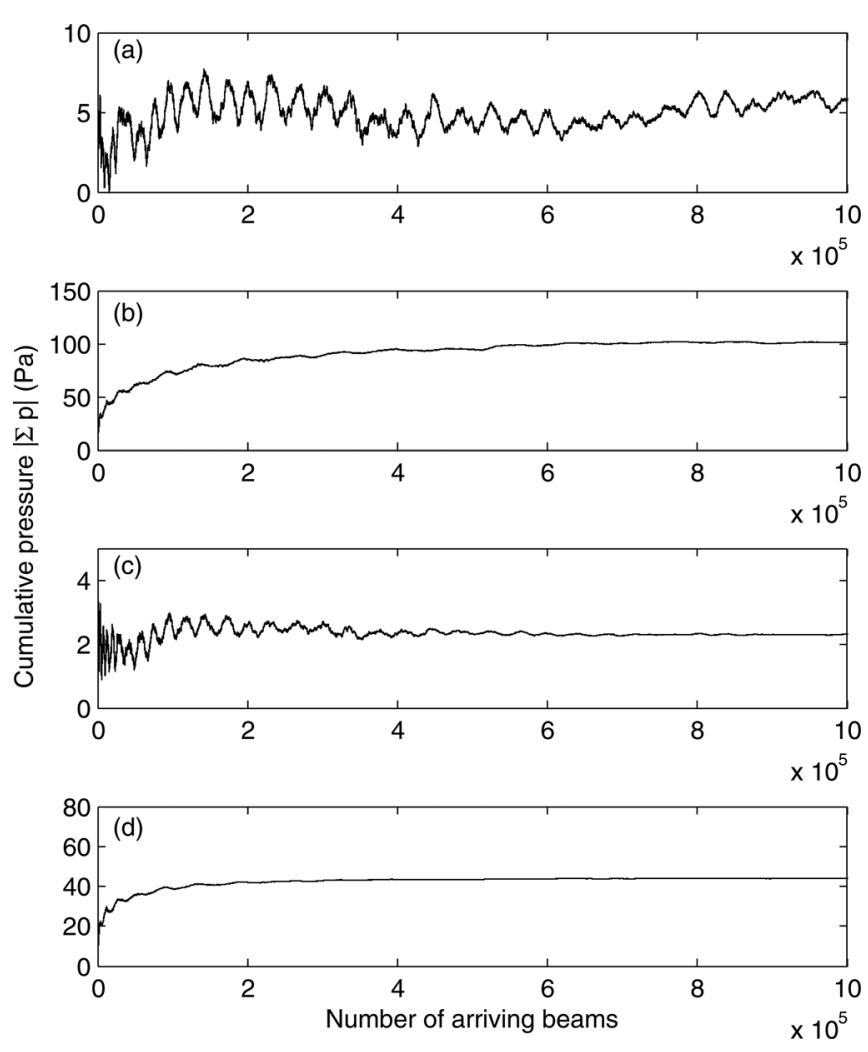

FIG. 2. Convergence of cumulative sound pressures: (a) and (c) $119 \mathrm{~Hz}$, (b) and (d) 968 Hz. (a) and (b) $\alpha=0.01$, (c) and (d) $\alpha=0.05$.

$$
G\left(f, r_{r} \mid r_{s}\right)=\frac{p\left(r_{r}\right)}{j \omega \rho Q} \approx-\frac{1}{V} \sum_{m} \frac{\psi_{m}\left(r_{r}\right) \psi_{m}\left(r_{s}\right)}{k^{2}-k_{m}^{2}-j k\left(\tau_{m} c\right)}
$$

where $p\left(r_{r}\right)$ is the sound pressure at $r_{r}, Q\left(=4 \pi a^{2} u_{a}\right)$ is the volume velocity of the monopole at $r_{s}, \omega$ is the angular frequency, $\rho$ is the density of air, $\tau_{m}$ is the time constant, and $c$ is the speed of sound. For each mode $N$ representing three integers $\left(n_{\mathrm{x}}, n_{\mathrm{y}}, n_{\mathrm{z}}\right)$,

$$
\psi_{N}\left(r_{r}\right)=\Lambda_{N} \cos \left(\frac{n_{x} \pi x}{l_{x}}\right) \cos \left(\frac{n_{y} \pi y}{l_{y}}\right) \cos \left(\frac{n_{z} \pi z}{l_{z}}\right)
$$

where $\Lambda_{N}$ denotes the normalization factor, $\sqrt{\varepsilon_{n_{x}} \varepsilon_{n_{y}} \varepsilon_{n_{z}}}$, in which $\varepsilon_{0}=1$, and $\varepsilon_{1}=\varepsilon_{2}=\varepsilon_{3}=\cdots=2$. Note that this specific formulation of Green's function is an approximation for small absorption and is valid only for uniform boundary conditions. The imaginary term in the denominator accounts for the room absorption, which is related to $T_{60}$ by $T_{60}=13.8$ $\tau_{m}$. Therefore the pressure predicted by Green's function is given by

$$
p_{\text {Green }}(f)=j \omega \rho Q \cdot G\left(f, r_{r} \mid r_{s}\right)=4 \pi G\left(f, r_{r} \mid r_{s}\right) .
$$

Once a transfer function by PBTM and $p_{\text {Green }}$ in Eq. (6) are calculated for a source-receiver pair, they are converted to sound pressure levels re $1 \mathrm{~Pa}$, viz., $S P L_{P B T M}$ and $S P L_{G r e e n}$, respectively, and compared to each other to quantify simulation errors.

\section{B. Boundary element method}

The boundary element method can solve acoustic problems numerically based on the discretized HelmholtzKirchhoff integral equation on a surface mesh. ${ }^{5}$ For thorough investigations of the effect of various uniform and nonuniform absorption distributions on the PBTM accuracy, an in-house boundary element model was used for a proportionate rectangular room (see Sec. III C 4). The room model has 3912 elements and 1958 nodes, therefore its upper frequency is about $700 \mathrm{~Hz}$. The linear shape function and seven Gaussian points were used.

\section{Test rooms and simulation conditions}

\section{Validation with Green's functions}

Narrow band spectra at $1 \mathrm{~Hz}$ intervals are mainly investigated for three rooms that are different in shape and volume: a unit cubic room, a well proportionate room with dimensions of $1.9 \times 1.4 \times 1 \mathrm{~m}$, a disproportionate room of dimensions of $5 \times 1 \times 1 \mathrm{~m}$ as shown in Fig. 3 . The second room ratio is based on Louden's work, which concluded that this room ratio is optimum for achieving evenly spaced modes. ${ }^{20}$ Therefore the first two rooms are the extremes in (a)

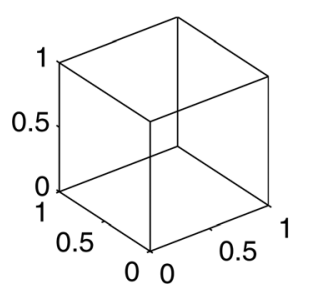

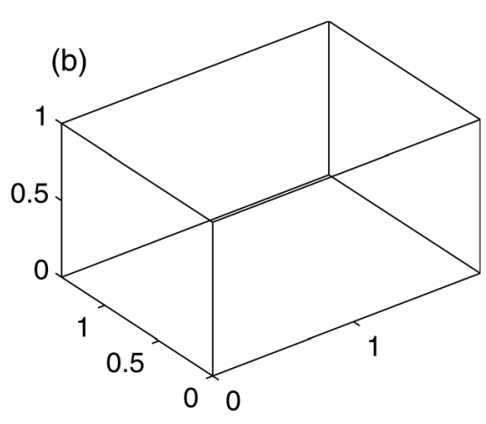

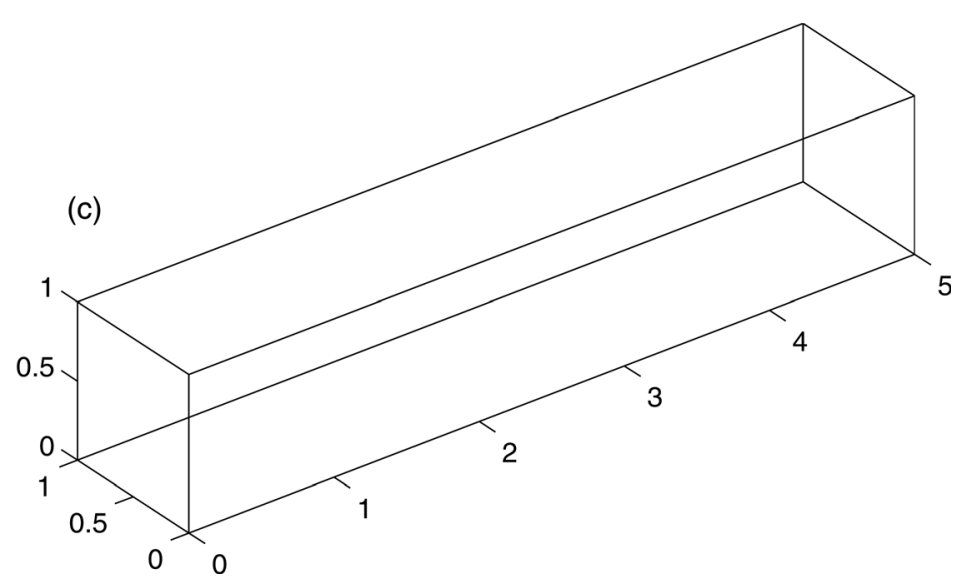

FIG. 3. Three tested rooms. (a) Unit cube, (b) proportionate room $(1.9 \times 1.4 \times 1 \mathrm{~m})$, (c) disproportionate $(5 \times 1 \times 1 \mathrm{~m})$. 
terms of the modal distribution because the unit cubic room has a large number of degenerate modes, whereas the room ratio of 1.9:1.4:1 assures an even distribution of the room modes. When the absorption coefficients of the boundary walls were assumed as 0.1 , the Schroeder frequencies of the three rooms were 1033, 732, and $540 \mathrm{~Hz}$, respectively. For all three rooms, nine sources and 20 receivers are chosen in each room for comparisons with Green's functions. Sound pressures are calculated in a frequency range from $20 \mathrm{~Hz}$ to the Schroeder frequency with steps of $1 \mathrm{~Hz}$, and simulated transfer functions are compared with the acoustic Green's functions for the same source and receiver. Additionally a larger cubic room with an edge length of $10 \mathrm{~m}$ is simulated to show that the simulation error is independent of the size of the room. For all the PBTM simulations, 8000 beams are emitted from the source, and they are traced up to the 100th reflection order. For example, in the cubic room where the mean free path is $0.67 \mathrm{~m}$, the mean propagation distance for the 100th reflection order is $67 \mathrm{~m}$, corresponding to a decay range of $46 \mathrm{~dB}$. In calculating Green's functions, acoustic modes up to $(100,100,100)$ are included using three cosine functions as the orthogonal modal shapes, $\psi_{m}$.

\section{Validation with BEM simulations}

The proportionate room $(1.9 \times 1.4 \times 1 \mathrm{~m})$ is further investigated with more realistic boundary conditions. First, various uniform boundary conditions were simulated. Specific surface impedances $(\zeta)$ of $40,30,20,10,7$, and 4 are used both for the boundary element model and for the PBTM simulations with the reflection modeling given by $r(\theta)=(\zeta \cos \theta-1) /(\zeta \cos \theta+1)$. The corresponding random incidence absorption coefficients are $0.17,0.20$, $0.30,0.49,0.60,0.79$, respectively. Note that the reflection coefficients used in the PBTM simulations are not complexvalued, but angle-dependent.

A realistic non-uniform absorption distribution was finally simulated to represent a typical lecture room condition up to the Schroeder frequency. The surface impedances for the ceiling, floor, and the side walls were 5.9, 18, and 38 , respectively, therefore the corresponding random absorption coefficients for the ceiling, floor, and side walls are $0.66,0.32$, and 0.17 , respectively. Note also that the surface impedances were used for PBTM and BEM simulations.

\section{Sources and receivers in the unit cube}

For the unit cube, nine source positions are defined. For each source, 20 receivers are located at the same height with increasing $d_{S R}$ at constant intervals. For example, receivers for the worst source located at $(0.5,0.5,0.5)$ are distributed along a diagonal line at $(x, x, 0.5)$, where $x$ ranges from 0.52 to 0.9 with steps of 0.02 . It is the worst source position because it coincides with nodal points, lines, and surfaces. One may then expect an extremely slow pressure convergence because only $12 \%$ of non-zero pressure amplitudes contribute to the total pressure. The other eight source positions are chosen so that they are not located close to either the boundary walls or the wellknown nodes: Their locations are $(0.1,0.1,0.5),(0.3,0.3$, $0.5),(0.1,0.1,0.2),(0.3,0.3,0.2),(0.15,0.25,0.1),(0.15$, $0.25,0.4),(0.35,0.45,0.4)$, and $(0.35,0.45,0.4)$. For each source of $\left(x_{0}, y_{0}, o\right)$, observation points are distributed along a diagonal line at $\left(x, y, z_{0}\right)$, where $x$ ranges from $x_{0}+0.02$ to $x_{0}+0.4$ with steps of 0.02 , and $y$ ranges from $y_{0}+0.02$ to $y_{0}+0.4$ with steps of 0.02 .

\section{Sources and receivers in the proportionate room}

For the proportionate room, nine source positions are defined. For each source, 20 receivers are located at the same height as the source with increasing $\mathrm{d}_{\mathrm{SR}}$ at uniform intervals. The source positions are $(0.1,0.05,0.1),(0.5,0.25$, $0.1),(1,0.5,0.1),(0.1,0.05,0.2),(0.5,0.25,0.2),(1,0.5$, $0.2),(0.1,0.05,0.35),(0.5,0.25,0.35)$, and $(1,0.5,0.35)$. Receivers for each source at $\left(x_{0}, x_{0} / 2, z_{0}\right)$ are distributed along a diagonal line at $\left(x, y, z_{0}\right)$, where $x$ ranges from $x_{0}+0.04$ to $x_{0}+0.8$ with steps of 0.04 , and $y$ ranges from $y_{0}+0.02$ to $y_{0}+0.4$ with steps of 0.02 .

This room is also used for realistic absorption boundary conditions because it can be regarded as a $1 / 4$ scale model of a lecture room. A teacher is assumed to stand at $(0.1,0.1$, 0.4 ), and students are distributed over a virtual surface of $z=0.3$. A total of 54 receivers are positioned with $x$ changing from 0.15 to 1.75 with steps of 0.2 and $y$ changing from 0.2 to 1.2 with steps of 0.2 .

\section{Sources and receivers in the disproportionate room}

For the disproportionate room, nine source positions are defined at $(0.7,0.14,0.1),(1.4,0.28,0.1),(2.1,0.42,0.1)$, $(0.7,0.14,0.2),(1.4,0.28,0.2),(2.1,0.42,0.2),(0.7,0.14$, $0.35),(1.4,0.28,0.35)$, and $(2.1,0.42,0.35)$. For each source, 20 receivers are distributed at the same height as the source with increasing $\mathrm{d}_{\mathrm{SR}}$. Receivers for each source at $\left(x_{0}\right.$, $\left.x_{0} / 5, z_{0}\right)$ are placed along a diagonal line at $\left(x, y, z_{0}\right)$, where $x$ ranges from $x_{0}+0.1$ to $x_{0}+2$ with steps of 0.1 , and $y$ ranges from $y_{0}+0.02$ to $y_{0}+0.04$ with steps of 0.02 .

\section{Error measures}

Three errors are estimated: an average error level over a narrow band spectrum from $20 \mathrm{~Hz}$ to the Schroeder frequency as $e_{1}$ in Eq. (7), an average error level over an 1/3 octave band spectrum as $e_{2}$ in Eq. (8), and a dissimilarity measure, $e_{3}$, using the modal assurance criterion (MAC) in Eq. (9). MAC implies a shape correlation of the calculated transfer functions by PBTM and Green's functions but note that $e_{3}$ is defined as 1-MAC, quantifying how dissimilar the two transfer functions are ${ }^{21}$

$$
\begin{aligned}
& e_{1}(d B)=\frac{1}{N_{\text {line }}} \sum_{i=20 \mathrm{~Hz}}^{\left\lfloor f_{\text {sch }}\right\rfloor}\left|\operatorname{SP} L_{P B T M}(i)-S P L_{\text {Green }}(i)\right| \\
& e_{2}(d B)=\frac{1}{N_{o b}} \sum_{n o b=1}^{N_{o b}} \mid S P L_{P B T M, o c t}(\text { nob })-S P L_{\text {Green }, \text { oct }}(\text { nob }) \mid
\end{aligned}
$$




$$
e_{3}=1-\frac{\left[S P L_{P B T M}(f)^{T} S P L_{\text {Green }}(f)\right]^{2}}{\left[S P L_{P B T M}(f)^{T} S P L_{P B T M}(f)\right] \cdot\left[S P L_{\text {Green }}(f)^{T} S P L_{\text {Green }}(f)\right]},
$$

where $f_{\text {sch }}$ is the Schroeder frequency of the room, $N_{\text {line }}$ is the number of frequency lines, that is, $\left\lfloor f_{s c h}\right\rfloor-19$, the symbol $\lfloor x\rfloor$ means the floor function, and $N_{o b}$ is the number of the $1 / 3$ octave bands below the Schroeder frequency. The same datasets are used for the narrow band error and dissimilarity of which the upper frequency is set to the Schroeder frequency.

If the two transfer functions are exactly the same, all errors should vanish. Because PBTM suffers from simulation noise mainly at off-resonance frequencies, $e_{1}$ is influenced by the simulation errors at off-resonance frequencies. A spectral shift may lead to a large $e_{1}$. The absorption of $10 \%$ assigned to the boundary surfaces naturally leads to shifts of the peaks and troughs toward lower frequency in the PBTM results, which can also affect $e_{1}$, in particular at the high end of the frequency range of interest.

The $1 / 3$ octave band error, $e_{2}$, is based on the sound pressure levels summed in $1 / 3$ octave bands. The lowest center frequency of the $1 / 3$ octave band is fixed to $31.5 \mathrm{~Hz}$, but the highest center frequency changes with the room: $800 \mathrm{~Hz}$ for the cube, $630 \mathrm{~Hz}$ for the proportionate room, and $400 \mathrm{~Hz}$ for the disproportionate room. The main causes of $e_{2}$ are differences in the sound pressures predicted near resonance frequencies, not the sound pressures at off-resonance frequencies. Therefore $e_{2}$ quantifies the accuracy of the PBTM simulations primarily at the resonance frequencies. Slight shifts of the peaks and troughs are not expected to affect $e_{2}$ significantly.

The dissimilarity measure, $e_{3}$, basically compares the shapes of the two spectra. The more similar the shapes are, the lower the dissimilarity. Note that the dissimilarity is most sensitive to the largest differences between the predicted transfer function by PBTM and acoustic Green's function and insensitive to small changes and/or small magnitudes. Shifts of the peaks and troughs may affect $e_{3}$ because it is affected by the sound pressures not only at the resonance frequencies but also at the off-resonance frequencies.

The errors are expected to change with the locations of source-receiver pairs. The main parameter might be $\mathrm{d}_{\mathrm{SR}}$ because the direct field is likely to overpower the reverberant field if they are very closely located, e.g., inside the room radius. ${ }^{22}$ As mentioned, the sources and receivers are chosen not too close to the boundary walls, e.g., less than $0.05 \mathrm{~m}$ to avoid additional simulation uncertainties.

\section{E. Analysis of variance}

Analysis of variance (ANOVA) tests have been applied to test if there are significant differences between the errors. ${ }^{23}$ For ANOVA tests, the commands "anova1" and "anova2" in MATLAB were used. A $p$ value from an ANOVA test is the chance of rejecting the null hypothesis when the null hypothesis is true. For smaller $p$ values than a signifi- cance level (normally 0.05 for * significance, 0.01 for ** significance, or 0.001 for $* * *$ significance), the null hypothesis is rejected, meaning there is a significant difference among the tested groups.

\section{RESULTS AND DISCUSSIONS}

\section{A. Changes in the aspect ratio with a uniform absorption of 0.1}

Figure 4 shows two example transfer functions for the first and fifth receiver in the cubic room between the PBTM results and Green's functions, when the source is placed at $(0.1,0.1,0.2)$. The smallest $e_{1}$ of $0.7 \mathrm{~dB}$ for the first receiver in Fig. 4(a) and the largest $e_{1}$ of $2.0 \mathrm{~dB}$ for the fifth receiver in Fig. 4(b) are found. The main causes of the smallest $e_{1}$ in Fig. 4(a) are the small deviations in the peak levels as well as the shifts of the peaks and troughs. However, the latter is actually not a PBTM error, but an inevitable consequence of the use of the three cosine terms in calculating the Green's functions, being an error in the Green's function. The largest error in Fig. 4(b) results mainly from noise contamination at the off-resonance frequencies, particularly in a frequency range from 600 to $800 \mathrm{~Hz}$. The PBTM simulations seem to be accurate when the predicted SPLs are higher than $10 \mathrm{~dB}$, which is approximately $30 \mathrm{~dB}$ below the maximum level. One may notice that the difference between the maximum and minimum SPL is only around $20 \mathrm{~dB}$ for the smallest error case, whereas the difference is much larger as $60 \mathrm{~dB}$ for the largest error case. Therefore Lam's $-30 \mathrm{~dB}$ cutoff level does not strictly hold for all phased geometrical acoustics simulations. ${ }^{2}$ Inaccurate predictions modify the sharpness of the troughs, leading to a large $e_{1}$. However, $e_{2}$ values
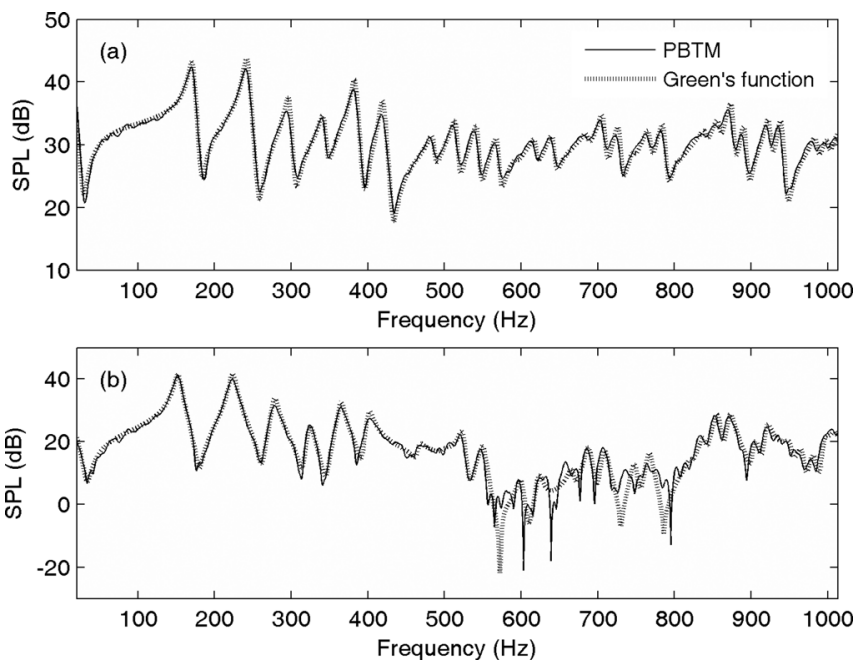

FIG. 4. Examples of predicted transfer function for a source $(0.1,0.1,0.2)$ in the cubic room. (a) Receiver at $(0.12,0.12,0.2)$, (b) receiver at $(0.2,0.2,0.2)$. 

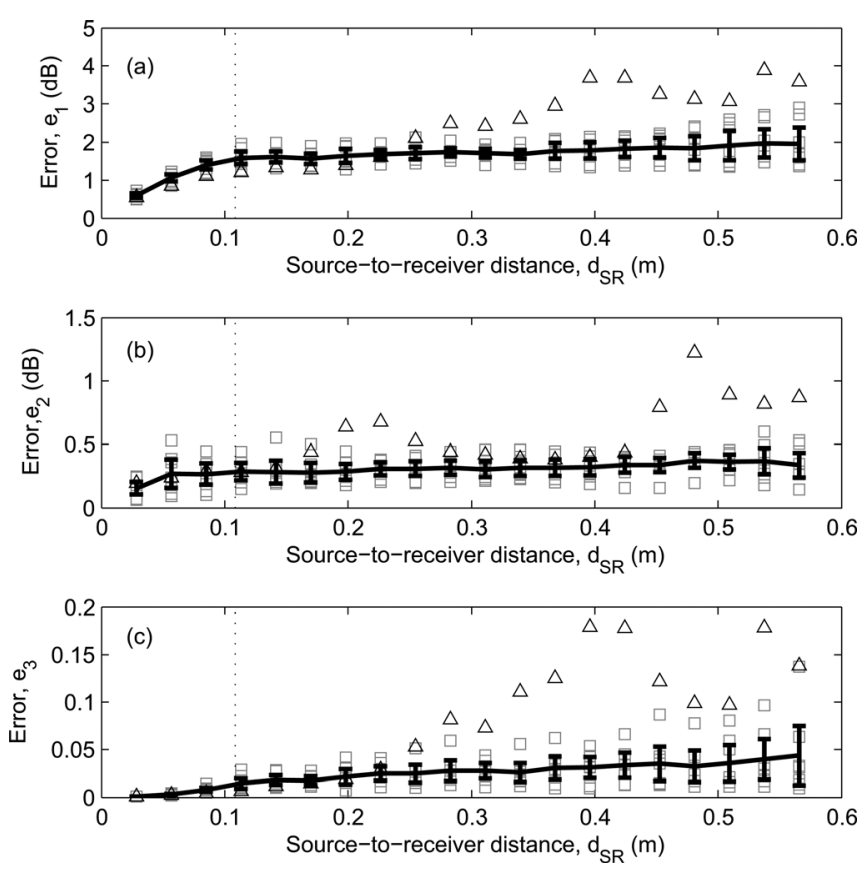

FIG. 5. Errors as a function of $d_{\mathrm{SR}}$ for the unit cube. $\triangle$ : Worst source case; $\square$ : The other eight sources; the line and error bars show the mean and $95 \%$ confidence intervals. (a) $e_{1}$, (b) $e_{2}$, (c) $e_{3}$. The dotted line indicates the room radius.

are quite similar and low as 0.2 and $0.4 \mathrm{~dB}$, respectively, which are not strongly influenced by the simulation errors at the off-resonance frequencies. The dissimilarity indices for the two receivers are found to be 0.001 and 0.03 , which are again significantly influenced by the simulation noise because the spectral shape of the transfer function changes near the off-resonance frequencies.

Figure 5 illustrates the errors as a function of $d_{S R}$ for the unit cube. The hollow triangles represent errors for the worst source at $(0.5,0.5,0.5)$, whereas the hollow square symbols are data points for the other eight sources. All the errors tend to be larger for the worst source. The means and $95 \%$ confidence intervals are plotted for the eight sources, indicating an increased error for a longer distance. For $e_{1}$, the error trend resembles the second-half of a logistic function in which the error increases linearly with the distance and saturated to a certain value. In this room, the mean value of $e_{1}$ is bounded to $2 \mathrm{~dB}$, indicating acceptable predictions. The $1 / 3$ octave band error $e_{2}$ is relatively independent of $\mathrm{d}_{\mathrm{SR}}$ and low enough to ensure accurately predicted SPLs in octave bands. The dissimilarity $e_{3}$ increases constantly as the distance increases, but the mean $e_{3}$ for the longest distance is still limited to 5\%. A two-way ANOVA shows that the $p$ values of $e_{1}$ are smaller than 0.001 for the receiver and source to conclude that they are significantly different in a statistical sense. However, the $p$ values of $e_{2}$ are high as 0.09 and 0.16 for the receiver and source, respectively; therefore one can conclude that $e_{2}$ does not change significantly with the source and receiver position. The dissimilarity $e_{3}$ is again statistically different because the $p$ values from a two-way ANOVA test are smaller than 0.001 for the receiver and source position, indicating $* * *$ significance $(p<0.001)$.
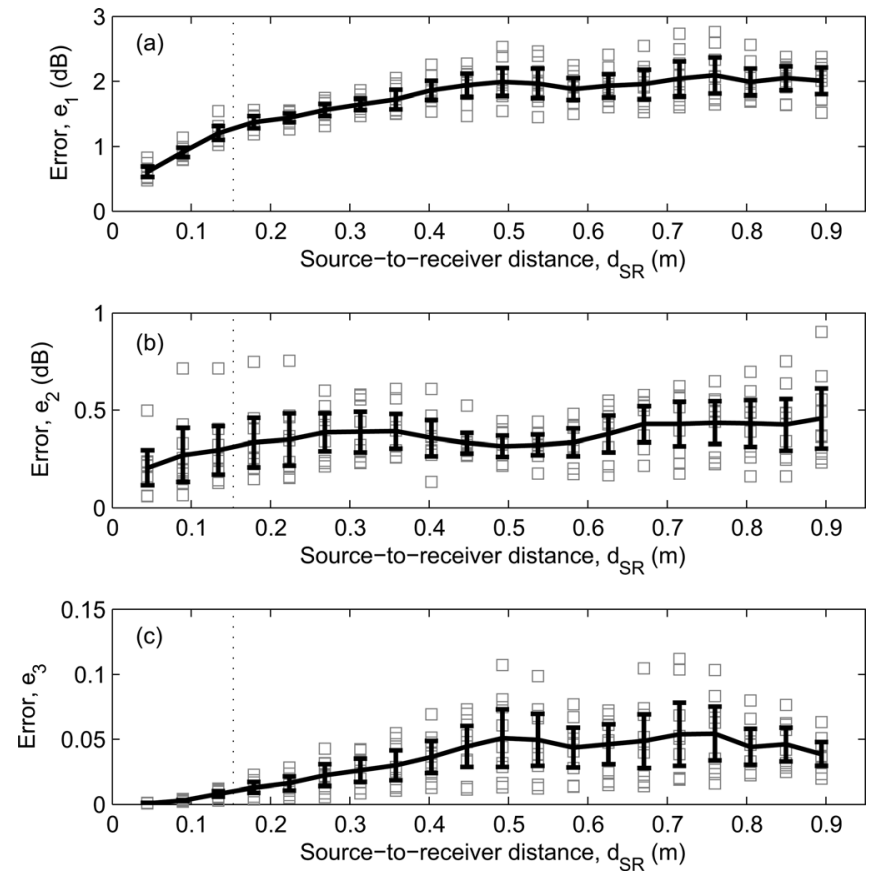

FIG. 6. Errors as a function of $\mathrm{d}_{\mathrm{SR}}$ for the proportionate room. $\square$ : Errors for the nine sources; the line and error bars show the mean and $95 \%$ confidence intervals. (a) $e_{1}$, (b) $e_{2}$, (c) $e_{3}$. The dotted line indicates the room radius.

Note that these ANOVA analyses do not include the worst source case.

The errors for the proportionate room are shown in Fig. 6, in which the trends are similar to the cubic room. The narrow band error $e_{1}$ is increased for longer distances but converged to around $2 \mathrm{~dB}$. The $1 / 3$ octave band error is not strongly affected by the distance, ranging from 0.2 to $0.5 \mathrm{~dB}$. The dissimilarity error $e_{3}$ is limited to 0.05 . Using an ANOVA test, $p$ values of $e_{1}$ are smaller than 0.001 for the receivers and sources, indicating $* * *$ significance. However, the $p$ value of $e_{2}$ is relatively high as 0.01 (** significance, $p<0.01$ ) for the receiver, while the $p$ value of $e_{2}$ for the source is small enough to confirm $* * *$ significance. The dissimilarity error $e_{3}$ is obviously statistically different because the $p$ values from a two-way ANOVA test are smaller than 0.001 for the receiver and source position.

The errors for the disproportionate room are shown in Fig. 7. The errors are larger than those for the other rooms. The narrow band error $e_{1}$ is bounded to $3 \mathrm{~dB}$. The $1 / 3$ octave band error $e_{2}$ increases with the distance, and its mean value for the longest distance becomes $1.8 \mathrm{~dB}$. The dissimilarity error $e_{3}$ is also increased up to 0.1. ANOVA tests show that all $p$ values of $e_{1}, e_{2}$, and $e_{3}$ are smaller than 0.001 for the receivers and sources, indicating $* * *$ significance. The errors investigated with changing the source and receiver positions are consistent for all the tested rooms. The narrow band error and dissimilarity increase and become constant after a certain distance. As expected, the $1 / 3$ octave band error is not strongly influenced by the simulation noise at the offresonance frequencies, making $e_{2}$ less dependent on $\mathrm{d}_{\mathrm{SR}}$.

Among the error measures, $e_{1}$ is correlated with $e_{3}$. For the unit cube, the coefficient of determination $\left(R^{2}\right)$ of a linear regression between $e_{1}$ and $e_{3}$ is 0.74 , whereas $R^{2}$ is only 0.29 

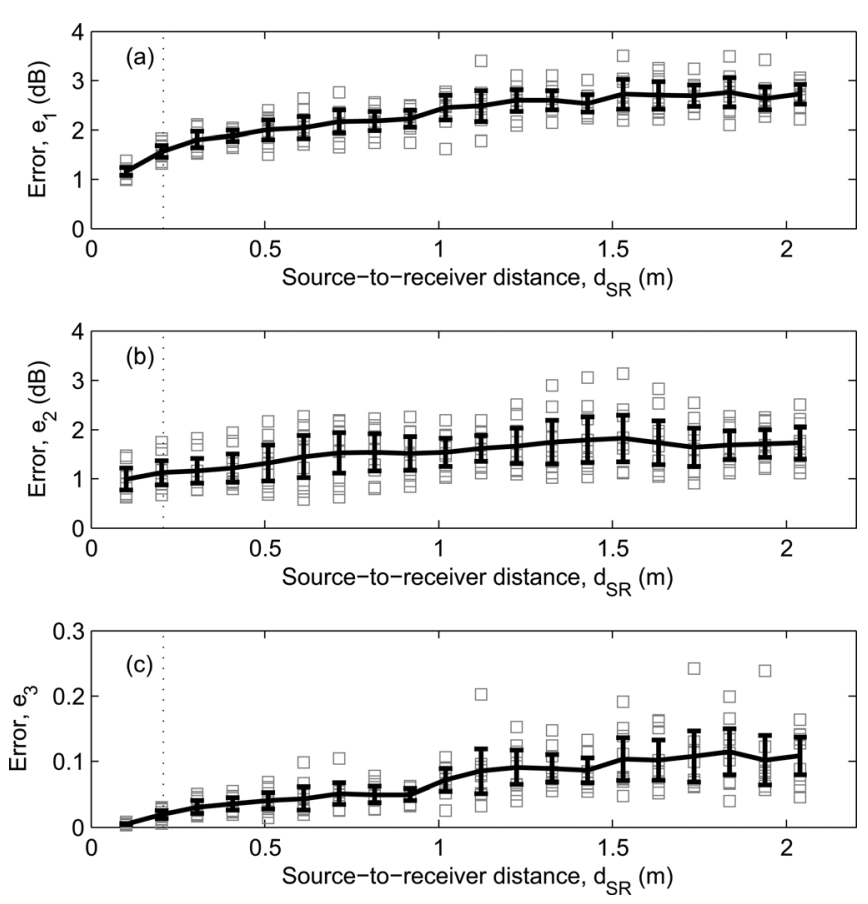

FIG. 7. Errors as a function of $\mathrm{d}_{\mathrm{SR}}$ for the disproportionate. $\square$ : Errors for the nine sources; the line and error bars show the mean and $95 \%$ confidence intervals. (a) $e_{1}$, (b) $e_{2}$, (c) $e_{3}$. The dotted line indicates the room radius.

between $e_{1}$ and $e_{2}$. For the proportionate room, $R^{2}$ values are 0.74 and 0.08 , respectively. Because $e_{2}$ is relatively independent of $\mathrm{d}_{\mathrm{SR}}$, whereas $e_{1}$ increases with increasing $\mathrm{d}_{\mathrm{SR}}$, the correlation between the two errors should be low. For the disproportionate, the coefficient of determination between $e_{1}$ and $e_{2}$ is somewhat higher as 0.44 , and the coefficient of determination between $e_{1}$ and $e_{3}$ is 0.86 . Because $e_{3}$ is strongly correlated with $e_{1}$, it can be regarded as a redundant error index.

It is assumed that the D-R energy ratio would change with $\mathrm{d}_{\mathrm{SR}}$. There is a well known concept of the room radius, at which the direct and reverberant energy are equal. For a diffuse sound field, it is calculated as $0.14 \sqrt{A},{ }^{22}$ where $A$ is the equivalent absorption area. For the three rooms, the room radius are $0.11,0.15,0.21$, respectively, indicated as the vertical dotted lines in Figs. 5-7. At least inside the room radius, both $e_{1}$ and $e_{3}$ increase linearly with $\mathrm{d}_{\mathrm{SR}}$.

The unit cube and proportionate room have similar error trends. Recalling that they are the extreme cases in terms of the modal distribution, the accuracy of PBTM simulations is consistent regardless of the distribution of the room mode. However, the errors are indeed affected by the aspect ratio of the room. The more disproportionate the room, the larger the errors. An increase in $e_{2}$ by about $1 \mathrm{~dB}$ for the disproportionate room implies that the predicted levels at the resonance frequencies are not as precise as in the other two rooms; this is associated with another simulation error due to different mean free paths. In the disproportionate room, there are a large variety of mean free paths: Beams traveling along the longest dimension of the enclosure have a longer mean free path than beams traveling across the space. Therefore the beams traveling along the longest dimension tend to diverge more and have a greater risk of the sampling error
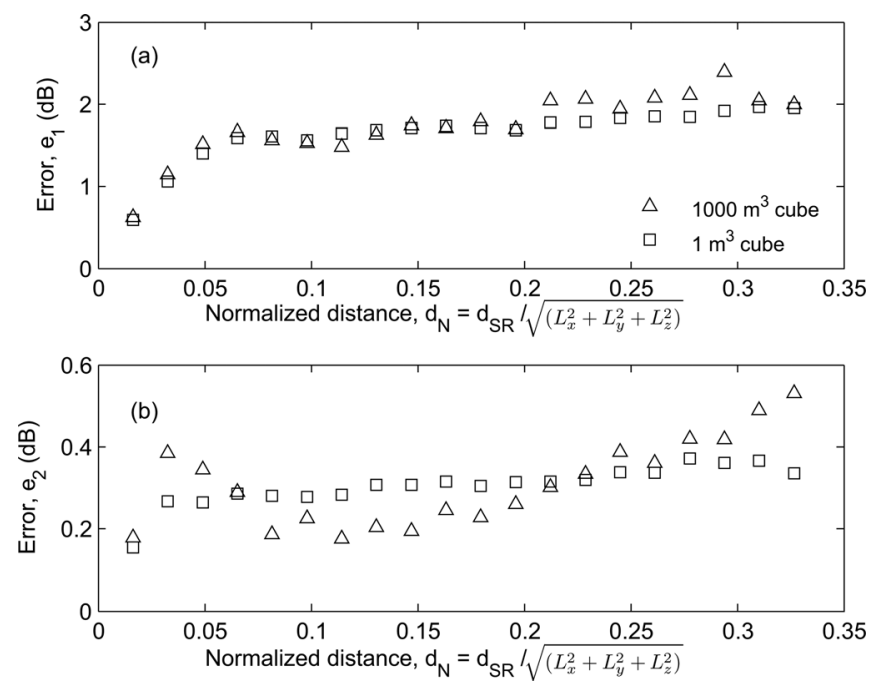

FIG. 8. Comparison of the errors between the unit cube and a cube with an edge of $10 \mathrm{~m}$. (a) $e_{1}$, (b) $e_{2}$.

because no splitting algorithm is employed in the current PBTM simulation. For example, the longest and shortest dimension of the disproportionate room correspond to the axial modes of 34 and $171 \mathrm{~Hz}$, respectively. The average level differences between PBTM result and acoustic Green's function at the two frequencies are 2.6 and $1 \mathrm{~dB}$, respectively, for the shortest $\mathrm{d}_{\mathrm{SR}}$, indicating that the beams along the longest dimension obviously suffer from a rather severe simulation noise. However, the level differences at the two axial modes gradually increase with increasing $\mathrm{d}_{\mathrm{SR}}$, ending up with similar values of 3.2 and $2.7 \mathrm{~dB}$, respectively, for the longest $\mathrm{d}_{\mathrm{SR}}$.

To test whether or not the room volume affect the PBTM simulation error, another cube with dimensions of $10 \times 10 \times 10 \mathrm{~m}$ was simulated and compared with the mean value of the unit cube in Fig. 8. In the larger cube, a source is located at $(1.5,2.5,1)$, and receivers are placed at $(x, y, 1)$ with $x$ changing from 1.7 to 5.5 at intervals of 0.2 and $y$ changing from 2.7 to 6.5 at intervals of 0.2 . For a fair comparison, the abscissa is a normalized distance, $\mathrm{d}_{\mathrm{N}}$, which is $\mathrm{d}_{\mathrm{SR}}$ divided by the maximum diagonal length of the room $\left(L_{\max }=\sqrt{L_{x}^{2}+L_{y}^{2}+L_{z}^{2}}\right)$. Although the room volume is increased by a factor of 1000 , the errors in Fig. 8 are not significantly increased, therefore the room volume does not change the simulation error significantly when the room geometry is the same.

An attempt to make an error model was carried out for $e_{1}$. In Fig. 9(a), one can observe the deviations in the mean $e_{1}$ values for the three rooms, which are mainly attributed to the aspect ratio. The errors for the disproportionate room are, on average, $0.5 \mathrm{~dB}$ higher than those for the unit cube, being equivalent to 1.12 times on the linear scale, whereas the errors for the proportionate room are virtually the same as those for the unit cube. Therefore one may assume a power-law function of $y=x^{b}$, as a simple correction, where $y$ is the incremental factor of $e_{1}$ compared with the cube (being 1.12 for the disproportionate room), and $x$ being the largest room dimension ratio (5 for the disproportionate room). 

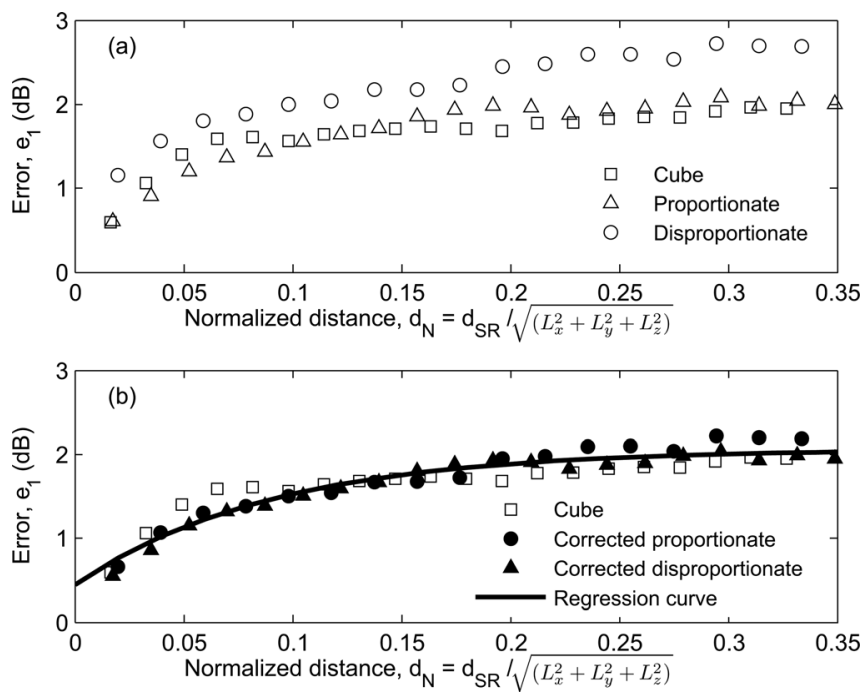

FIG. 9. Mean $e_{1}$ as a function of the normalized distance. (a) Uncorrected errors, (b) corrected errors.

Then the exponent $b$ is found to be 0.0715. Applying this correction to the proportionate room, where the largest room dimension ratio of 1.9 , ends up with an incremental factor of 1.011 , converting to $0.05 \mathrm{~dB}$ on the log scale. The corrected errors for the proportionate room and disproportionate are plotted in Fig. 9(b) as the solid symbols. A logistic function is used for fitting the error as follows:

$$
e_{1}(d B)=-A_{1}+\frac{A_{2}}{\left(1+\exp \left(-\left(\mathrm{d}_{\mathrm{N}}-A_{3}\right) / A_{4}\right)\right)} .
$$

The coefficients $\left(A_{1}, A_{2}, A_{3}\right.$, and $\left.A_{4}\right)$ for the regression line in Fig. 9(b) and its $R^{2}$ are listed in Table I. For $e_{2}$, it is not worth developing such a model because it is weakly dependent on $\mathrm{d}_{\mathrm{SR}}$.

One may ask a question if there is a significant difference in the errors among the rooms. A one-way ANOVA using the mean error values for the three test rooms, $p$ values are low as $2 \times 10^{-6}, 2 \times 10^{-35}, 1 \times 10^{-7}$ for $e_{1}, e_{2}$, and $e_{3}$, respectively, meaning that the errors are statistically significantly different depending on the room. However, the differences between the cubic and proportionate room are relatively small for which $p$ values are high as 0.6, 0.003, and 0.07 for $e_{1}, e_{2}$, and $e_{3}$, respectively. Only $e_{2}$ shows $* *$ significance, whereas the others are not significantly different between the two rooms. However, note that $d_{N}$ is not exactly the same for the three rooms as can be seen in Fig. 9, therefore this analysis may not be strictly correct although it is likely.

\section{B. Various uniform absorption cases}

The simulation errors, $e_{1}$ and $e_{2}$, due to different but uniformly distributed absorption are shown in Fig. 10. From the

TABLE I. Coefficients for regressions and corresponding coefficients of determination $\left(R^{2}\right)$.

\begin{tabular}{lrrrrr}
\hline \hline & \multicolumn{1}{c}{$A_{1}$} & \multicolumn{1}{c}{$A_{2}$} & \multicolumn{1}{c}{$A_{3}$} & $A_{4}$ & $R^{2}$ \\
\hline Regression 1 for the fixed absorption & -508.81 & 510.87 & -0.52 & 0.09 & 0.91 \\
Regression 2 for various absorption cases & 0.32 & 0.92 & 0.10 & 0.13 & 0.66 \\
\hline \hline
\end{tabular}
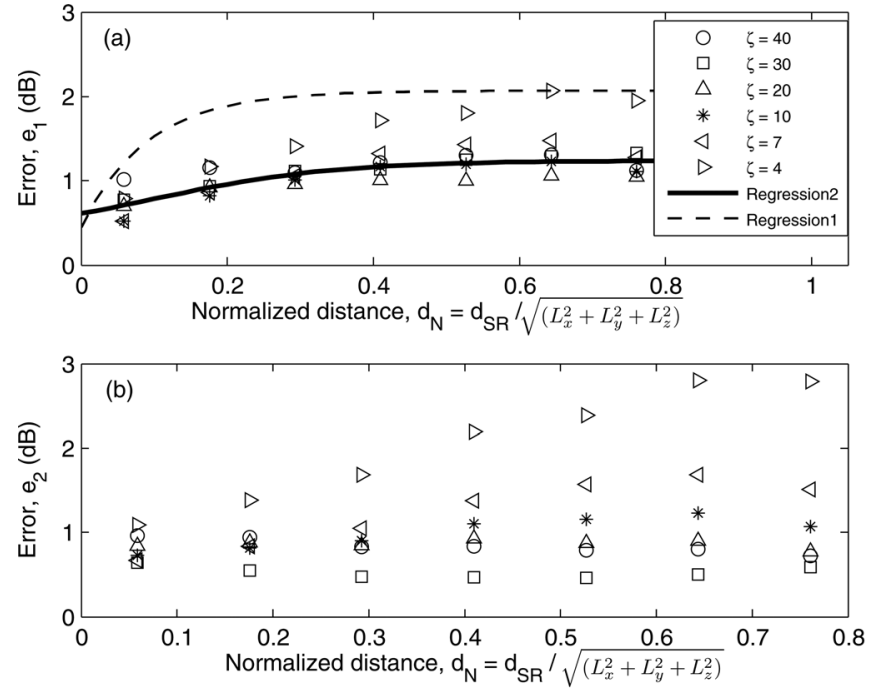

FIG. 10. Simulation errors as a function of the normalized distance for the proportionate room with uniform absorption distribution. (a) $e_{1}$, (b) $e_{2}$.

54 source-receiver pairs having irregular $\mathrm{d}_{\mathrm{SR}}$, a new set of $\mathrm{d}_{\mathrm{SR}}$ is computed at intervals of $0.3 \mathrm{~m}$ from $0 \mathrm{~m}$ to $2 \mathrm{~m}$, and the errors within each interval are averaged. The narrow band error seems to increase with $\mathrm{d}_{\mathrm{SR}}$ but converged to a certain value as can be seen in Fig. 10(a). For the first four cases having absorptions lower than 0.5 , the narrow band errors are similar with a small deviation, whereas the largest $e_{1}$ is found for the highest absorption case as also can be seen in Table II. The smallest and largest $e_{1}$ are 1.0 and $1.7 \mathrm{~dB}$ for the cases 3 and 6 , respectively, therefore the maximum deviation in $e_{1}$ is $0.7 \mathrm{~dB}$.

The $1 / 3$ octave band errors are shown in Fig. 10(b). The $1 / 3$ octave band errors differ by $1.7 \mathrm{~dB}$ by changing the absorption condition in Table II, which is larger than the deviation in $e_{1}$. The maximum $e_{2}$ is $2.2 \mathrm{~dB}$ for case 6 , whereas the minimum error is $0.5 \mathrm{~dB}$ for a low absorption. For the first four cases, $e_{2}$ is constant over $\mathrm{d}_{\mathrm{SR}}$, but $e_{2}$ increases with increasing $\mathrm{d}_{\mathrm{SR}}$ for the last two absorption cases.

For the first four cases, $e_{1}$ is larger than $e_{2}$, while the opposite is true for the highest absorption case. When the simulation error near the off-resonance frequencies is predominant, $e_{2}$ is naturally smaller than $e_{1}$, as can be seen in Sec. IV A. A larger $e_{2}$ than $e_{1}$ implies two possibilities: The predicted peak levels are imprecise and/or the errors at low frequencies are larger because $e_{2}$ is based on $1 / 3$ octave

TABLE II. Errors for six uniform absorption conditions in the proportionate room. The surface impedance, corresponding normal and random incidence coefficients, and Schroeder frequencies are indicated.

\begin{tabular}{lccccccc}
\hline \hline & $\zeta$ & $\alpha_{\text {nor }}$ & $\alpha_{\text {rand }}$ & $f_{\text {Sch }}$ & Mean $e_{1}$ & Mean $e_{2}$ & Mean $e_{3}$ \\
\hline Case 1 & 40 & 0.10 & 0.17 & 566 & 1.2 & 0.8 & 0.02 \\
Case 2 & 30 & 0.13 & 0.20 & 520 & 1.2 & 0.5 & 0.02 \\
Case 3 & 20 & 0.18 & 0.30 & 423 & 1.0 & 0.9 & 0.01 \\
Case 4 & 10 & 0.33 & 0.49 & 331 & 1.1 & 1.0 & 0.01 \\
Case 5 & 7 & 0.43 & 0.60 & 299 & 1.3 & 1.3 & 0.02 \\
Case 6 & 4 & 0.64 & 0.79 & 260 & 1.7 & 2.2 & 0.04 \\
Non-uniform & & 0.21 & 0.31 & 414 & 2.2 & 1.5 & 0.04 \\
\hline \hline
\end{tabular}


TABLE III. $e_{1}$ as a function of the center frequency in the proportionate room.

\begin{tabular}{lcccccccccc}
\hline \hline$f_{c}(\mathrm{~Hz})$ & 31.5 & 40 & 50 & 63 & 80 & 100 & 125 & 160 & 200 & 250 \\
\hline Case 1 & 0.4 & 0.5 & 0.6 & 0.7 & 1.2 & 1.3 & 1.2 & 1.1 & 1.1 & 1.2 \\
Case 2 & 0.3 & 0.4 & 0.5 & 0.5 & 0.6 & 0.5 & 0.6 & 0.6 & 1.0 & 1.0 \\
Case 3 & 0.6 & 0.6 & 0.7 & 0.8 & 1.6 & 1.5 & 1.3 & 1.1 & 1.0 & 1.1 \\
Case 4 & 1.3 & 1.1 & 1.0 & 1.1 & 1.9 & 1.8 & 1.3 & 1.2 & 1.0 & 1.0 \\
Case 5 & 2.1 & 1.6 & 1.4 & 1.5 & 2.1 & 1.9 & 1.4 & 1.2 & 1.0 & 1.0 \\
Case 6 & 4.4 & 3.4 & 2.9 & 2.7 & 2.5 & 1.9 & 1.5 & 1.0 & 0.8 & 0.7 \\
Non-uniform & 0.7 & 0.7 & 0.9 & 1.0 & 1.0 & 1.9 & 1.8 & 1.8 & 2.3 & 2.4 \\
\hline \hline
\end{tabular}

band levels, therefore has larger weightings at lower frequencies. Table III shows $e_{1}$ as a function of frequency, where the case 6 has large errors at frequencies lower than $125 \mathrm{~Hz}$. It is due mainly to the spherical error, which is also briefly discussed in Ref. 2, for highly absorbing walls at low frequencies, say, below $100 \mathrm{~Hz}$, because the PBTM simulations employed the plane wave reflection coefficient. As frequency increases, the sphericity error is reduced as shown in Table III. For high absorptions, the troughs are not as sharp as for the low absorptions, therefore the simulation error near off-resonance frequencies are unlikely to dominate, resulting in smaller errors except at very low frequencies. Note that the error for the case 6 in the $250 \mathrm{~Hz}$ band is 0.7 , which is actually smaller than that for the case 1.

Because the highest absorption case shows a large deviation with the others in Fig. 10(a), case 6 was excluded when developing a regression model. The coefficients for the regression model of $e_{1}$ for the various absorption cases and its $R^{2}$ are listed in Table I. Note that this regression model is valid for a random incidence absorption coefficient from 0.2 to 0.6. Compared to the regression model for the fixed absorption of 0.1 , one can observe lower errors with the boundary element validations, because the Green's functions inherently include certain errors as mentioned in the first paragraph in Sec. IV A. The narrow band error is limited to $1.3 \mathrm{~dB}$ for absorption coefficients lower than 0.6 , which are reasonable scenarios at low frequencies.

\section{Non-uniform absorption case}

Because the proportionate room is regarded as a scaled classroom, a realistic non-uniform distribution has been tested. The random incidence absorption coefficients of the ceiling, floor, and side walls are $0.66,0.32$, and 0.17 , respectively, leading the mean absorption coefficient to 0.31 , which is similar to the uniform absorption case 3. $e_{1}$ and $e_{2}$ for the non-uniform absorption case are shown in Fig. 11: $e_{1}$ changes with the source-to-receiver distance, whereas $e_{2}$ is relatively constant over $d_{S R}$. The room radius of $0.27 \mathrm{~m}$ is indicated as the dotted line.

The mean $e_{1}, e_{2}$, and $e_{3}$ are $2.2 \mathrm{~dB}, 1.5 \mathrm{~dB}$, and $0.05 \mathrm{~dB}$, respectively. Compared to the uniform absorption case $3, e_{1}$ and $e_{2}$ are increased by 1.2 and $0.6 \mathrm{~dB}$, respectively, due entirely to the non-uniform distribution of absorption. In Table III, noticeable increases in the error are observed at the higher frequencies close to the Schroder frequency. The

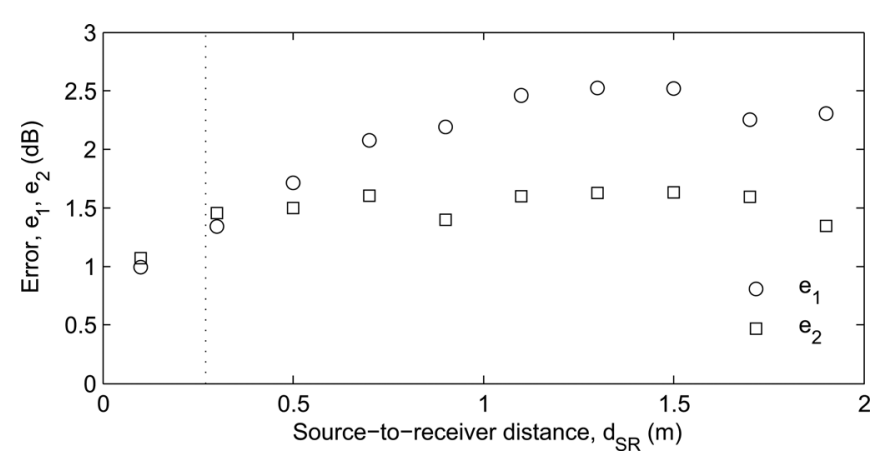

FIG. 11. Simulation errors as a function of the source-to-receiver distance for the rectangular room with non-uniform absorption distribution. The dotted line represents the room radius of $0.27 \mathrm{~m}$.

reason might be that the phased beam tracing used cannot account for wave phenomena due to the discontinuity in the boundary condition, i.e., wave diffraction and scattering evoked by the discontinuity. Lam speculated that the boundaries between the absorption changes will diffract sound, therefore the simulation accuracy might be reduced for a non-uniform distribution of absorption. ${ }^{2}$ A relevant theory for such discontinuities of the surface impedance (or admittance) has been derived by Morse and Ingard for a 2D case ${ }^{12}$ and later used by Thomasson. ${ }^{24}$ They assumed a finite absorber located on an acoustically hard plane and examined the sound pressure distribution over the absorber by a variational approach. In their derivations, the sound pressure over the absorber is assumed to be a summation of the incident wave, its specularly reflected component, and an unknown scattered component due to the impedance-discontinuous boundary. Thomasson used this formulation to account for overestimated statistical absorption coefficients measured in a reverberation chamber. ${ }^{23}$ Similar investigations can be attempted for non-uniform distributions of absorption in rooms, particularly how the pressure distribution changes near impedance-discontinuous 3D boundaries. From such investigations, an advanced reflection modeling can be developed and used to enhance the simulation accuracy.

All the errors are limited to $3 \mathrm{~dB}$ for all the tested geometries and absorption cases; this implies that PBTM is capable of predicting the sound pressures except for absorption cases lower than 0.1 at low frequencies. Therefore PBTM can be a viable acoustic simulation method for calculating narrow or $1 / 3$ octave band levels at frequencies even below the Schroeder frequency in rectangular rooms. The simulation error is reduced for well proportionate and/or moderately damped rooms with short source-to-receiver distances but increased for higher absorption, long distances, and nonuniform distributions of wall absorption.

\section{OUTLOOK}

The phased beam tracing method has potential applications such as small-to-medium sized lecture/conference rooms and studios where wave-related problems occur mainly at frequencies lower than the Schroeder frequency. Simulations using the phased beam tracing are much faster than finite/boundary element simulations. For example, a 
narrow band spectrum calculation at $2 \mathrm{~Hz}$ intervals from 20 to $1000 \mathrm{~Hz}$ using 8000 beams up to the 100th order of the $1000 \mathrm{~m}^{3}$ cubic room on a $2.4 \mathrm{GHz}$ Core2 Duo CPU with a 4 GB RAM, it takes only $1.5 \mathrm{~h}$ while a boundary element calculation takes $56.5 \mathrm{~h}$. Note, however, that both simulation codes are not particularly optimized for speeding up calculations. For PBTM to be used in practice, advanced algorithms for diffraction and diffuse reflection should be incorporated. The simulation accuracy using different types of boundary conditions, namely, absorption coefficients and surface impedances still needs to be more investigated. The advanced reflection modeling discussed in Sec. IVC for absorption/impedance-discontinues boundaries will further enhance the simulation accuracy.

\section{CONCLUSIONS}

The accuracy of a phased beam tracing method has been investigated by changing the locations of sources and receivers in three rectangular rooms with different absorption and aspect ratios. The narrow band error averaged from $20 \mathrm{~Hz}$ to the Schroeder frequency increases with the source-receiver distance but limited to a certain value. For a uniform absorption of 0.1 with various aspect ratios of rectangular rooms, the maximum narrow band errors are around $2 \mathrm{~dB}$ for the unit cube and proportionate room and 3 $\mathrm{dB}$ for the disproportionate room, which reveals that the error is affected by the aspect ratio of the room. The $1 / 3$ octave band error does not depend strongly on the $\mathrm{d}_{\mathrm{SR}}$, being less than $1 \mathrm{~dB}$ for the cubic and proportionate room and $2 \mathrm{~dB}$ for the disproportionate room. The dissimilarity index does vary with the source-receiver distance in a similar way with the narrow band error. The PBTM simulation error is influenced by the room absorption in the proportionate room within a maximum deviation of around 1.7 $\mathrm{dB}$. Interestingly, the minimum error is found for a random incidence absorption coefficient of between 0.3 and 0.5 . For absorptions lower than 0.3, the errors near the off-resonance frequencies dominate, therefore yielding larger narrow band errors. On the other hand, the sphericity error becomes severe for absorptions higher than 0.6 at the very low frequencies below $100 \mathrm{~Hz}$. For all the investigated absorption conditions, the simulation error does not exceed $2.2 \mathrm{~dB}$. For a realistic non-uniform distribution of wall absorption, the errors are increased by approximately $1 \mathrm{~dB}$ due to the wave phenomena evoked by the discontinuity in the boundary conditions, requiring more advanced reflection modeling methods for accurate predictions. All in all, the accuracy of PBTM for the well proportionate rectangular room is found to be quite acceptable, resulting in the maximum $1 / 3$ octave band error of $2.2 \mathrm{~dB}$ in the low frequency range below the Schroeder frequency. Therefore it is concluded that PBTM is suitable for calculating sound pressure spectra in rectangular rooms even below the Schroeder frequency, and its accuracy is enhanced for shorter source-receiver distances in more proportionate and moderately damped rooms.

\section{ACKNOWLEDGMENTS}

This research was partially supported by the BK21 project and the NCRC grant (NRF 2011-0018242).

${ }^{1}$ J. S. Suh, and P. A. Nelson, "Measurement of transient response of rooms and comparison with geometrical acoustic models," J. Acoust. Soc. Am. 105, 2304-2317 (1999).

${ }^{2}$ Y. W. Lam, "Issues for computer modelling of room acoustics in nonconcert hall settings,” Acoust. Sci. Tech. 26, 145-155 (2005).

${ }^{3}$ C.-H. Jeong, J.-G. Ih, and J. H. Rindel, "An approximate treatment of reflection coefficient in the phased beam tracing method for the simulation of enclosed sound fields at medium frequencies," Appl. Acoust. 69, 601-613 (2008).

${ }^{4} \mathrm{M}$. Hodgson, and A. Wareing, "Comparisons of predicted steady-state levels in rooms with extended- and local-reaction bounding surfaces," J. Sound Vib. 309, 167-177 (2008).

${ }^{5}$ M. Vorlander, Auralization: Fundamentals of Acoustics, Modelling, Simulation, Algorithms and Acoustic Virtual Reality (Springer-Verlag, Berlin, 2008), p. 155.

${ }^{6}$ M. Toyama, H. Suzuki, and Y. Ando, The Nature and Technology of Acoustic Space (Academic, Tokyo, 1995), p. 80.

${ }^{7}$ E. D. Geest and H. Patzold, "Comparison between room transmission functions calculated with a boundary element method and a ray tracing method including phase," in Proceedings of Inter-Noise 96, Liverpool, UK (1996), pp. 3177-3180.

${ }^{8}$ A. Wareing and M. Hodgson, "Beam-tracing model for predicting sound field in rooms with multilayer bounding surfaces," J. Acoust. Soc. Am. 118, 2321-2331 (2005).

${ }^{9}$ R. G. Kouyoumjian and P. H. Pathak, "A uniform geometrical theory of diffraction for an edge in a perfectly conducting surface," Proc. IEEE 62, 1448-1461 (1974).

${ }^{10}$ N. Tsingos, I. Carlbom, G. Elko, R. Kubli, and T. Funkhouser, "Validating acoustical simulations in the Bell Labs box," IEEE Comput. Graphics Appl. 22, 28-37 (2002).

${ }^{11} \mathrm{~T}$. Lewers, "A combined beam tracing and radiant exchange computermodel of room acoustics," Appl. Acoust. 38, 161-178 (1993).

${ }^{12}$ P. M. Morse and K. U. Ingard, Theoretical Acoustics (McGraw-Hill, New York, 1968), Chap. 8.4, pp. 310, 555.

${ }^{13}$ A. D. Pierce, Acoustics. An Introduction to Its Physical Principles and Applications (AIP, Woodbury, NY, 1991), Chap. 9.

${ }^{14}$ N. Tsingos, T. Funkhouser, A. Ngan, and I. Carlbom, "Modeling acoustics in virtual environments using the uniform theory of diffraction," in Proceedings of ACM SIGGRAPH, (2001), CD-ROM.

${ }^{15}$ R. R. Torres, U. P. Svensson, and M. Kleiner, "Computation of edge diffraction for more accurate room acoustics auralization," J. Acoust. Soc. Am. 109, 600-610 (2001).

${ }^{16}$ T. Funkhouser, N. Tsingos, I. Carlbom, G. Elko, M. Sondhi, J. E. West, G. Pingali, P. Min, and A. Ngan, "A beam tracing method for interactive architectural acoustics,” J. Acoust. Soc. Am. 115, 739-756 (2004).

${ }^{17}$ I. A. Drumm and Y. W. Lam, "The adaptive beam-tracing algorithm," J. Acoust. Soc. Am. 107, 1405-1412 (2000).

${ }^{18}$ D. Maercke and J. Martin, "The prediction of echograms and impulse responses within the Epidaure software," Appl. Acoust. 38, 93-114 (1993).

${ }^{19}$ T. Sakuma, S. Sakamoto, and T. Otsuru, "A benchmarking framework for wave-based computational methods in architectural acoustics," in Proceedings of ICA 2004, Kyoto, Japan (2004).

${ }^{20}$ M. M. Louden, "Dimension ratios of rectangular rooms with good distribution of eigentones," Acustica 24, 101-104 (1971).

${ }^{21}$ R. J. Allemang and D. L. Brown, "A correlation coefficient for modal vector analysis," in Proceedings of the 1st International Modal Analysis Conference (1982), pp. 110-116.

${ }^{22}$ H. Kuttruff, Room Acoustics, 4th ed. (Spon Press, London, 2000), p. 136.

${ }^{23}$ M. Kutner, C. Nachtsheim, and J. Neter, Applied Linear Statistical Model, 4th ed. (McGraw Hill, New York, 2004), Chap. 18.

${ }^{24}$ S.-I. Thomasson, "On the absorption coefficient," Acustica 44, 265-273 (1980). 